\title{
Determining Corticospinal Tract Injury from Stroke Using Computed Tomography
}

\author{
Timothy K. Lam, Daniel K. Cheung, Seth A. Climans (D), Sandra E. Black, \\ Fuqiang Gao, Gregory M. Szilagyi, George Mochizuki, Joyce L. Chen
}

\begin{abstract}
Introduction: Damage to the corticospinal tract (CST) from stroke leads to motor deficits. The damage can be quantified as the amount of overlap between the stroke lesion and CST (CST Injury). Previous literature has shown that the degree of motor deficits post-stroke is related to the amount of CST Injury. These studies delineate the stroke lesion from structural T1-weighted magnetic resonance imaging (MRI) scans, often acquired for research. In Canada, computed tomography (CT) is the most common imaging modality used in routine acute stroke care. In this proof-of-principle study, we determine whether CST Injury, using lesions delineated from CT scans, significantly explains the variability in motor impairment in individuals with stroke. Methods: Thirty-seven participants with stroke were included in this study. These individuals had a CT scan within the acute stage (7 days) of their stroke and underwent motor assessments. Brain images from CT scans were registered to MRI space. We performed a stepwise regression analysis to determine the contribution of CST injury and demographic variables in explaining motor impairment variability. Results: Using clinically available CT scans, we found modest evidence that CST Injury explains variability in motor impairment $\left(R_{\text {adj }}^{2}=0.12, p=0.02\right)$. None of the participant demographic variables entered the model. Conclusion: We show for the first time a relationship between CST Injury and motor impairment using CT scans. Further work is required to evaluate the utility of data derived from clinical CT scans as a biomarker of stroke motor recovery.
\end{abstract}

RÉSUMÉ : Déterminer l'étendue des lésions de la voie corticospinale à la suite d'un AVC au moyen d'un examen de tomodensitométrie. Introduction : Des lésions de la voie corticospinale à la suite d'un AVC vont provoquer des déficits moteurs. Ces lésions peuvent être quantifiées en observant le nombre de chevauchements (overlap) entre les lésions produites par un AVC et les lésions de la voie corticospinale. Des publications scientifiques antérieures ont en effet montré que le degré de déficits moteurs post-AVC est lié à la gravité des lésions de la voie corticospinale. Ces publications ont pu délimiter l'étendue des lésions des AVC au moyen d'examens d'IRM pondérés en T1, les appareils ayant été souvent acquis à des fins de recherche. Au Canada, la tomodensitométrie est la modalité d'IRM la plus couramment utilisée dans les soins de routine prodigués à la suite d'un AVC aigu. Dans cette étude de preuve de concept (proof-of-principle study), nous avons voulu déterminer dans quelle mesure les lésions de la voie corticospinale peuvent expliquer de manière satisfaisante la variabilité en termes de déficit moteur qu'on observe chez les individus victimes d'un AVC. Méthodes : Au total, 37 participants ont été inclus dans notre étude. Ces derniers ont subi un examen de tomodensitométrie dans la phase aiguë (7 jours) de leur AVC et ont également fait l'objet d'évaluations de leurs fonctions motrices. Les images de leur cerveau ont été captées dans un environnement de type IRM. Nous avons ensuite effectué une analyse de régression par étapes pour déterminer l'impact des lésions de la voie corticospinale et de variables démographiques dans la variabilité des déficits moteurs. Résultats : À l'aide d'examens de tomodensitométrie, nous avons donc trouvé des preuves limitées que les lésions de la voie corticospinale peuvent expliquer la variabilité des déficits moteurs $\left(\mathrm{R}^{2}{ }_{\text {ajusté }}=0,12 ; p=0,02\right)$. À noter qu'aucune des variables démographiques des participants n'a été incluse dans ce modèle. Conclusion : À l'aide d'examens de tomodensitométrie, nous avons ainsi montré pour la première fois qu'il existe une relation entre les lésions de la voie corticospinale et les déficits moteurs. De plus amples travaux sont toutefois nécessaires pour évaluer l'utilité, à titre de biomarqueur du rétablissement moteur à la suite d'un AVC, des données obtenues avec ces appareils de tomodensitométrie.

Keywords: Motor, Corticospinal tract, Computed tomography, Magnetic resonance imaging, Stroke

doi: $10.1017 / \operatorname{cjn} .2020 .112$

Can J Neurol Sci. 2020; 47: 775-784

\section{INTRODUCTION}

Neurological biomarkers derived from neuroimaging enable clinicians to differentiate between patients with similar clinical presentations and determine their recovery potential. ${ }^{1-3}$ In particular, the damage to the corticospinal tract (CST) has been extensively studied with transcranial magnetic stimulation (TMS) and/or magnetic resonance imaging (MRI) as a biomarker of stroke motor impairment. ${ }^{4-13}$ For example, the Predict Recovery Potential-2 algorithm ${ }^{14}$ uses a combination of clinical measures and neurological biomarkers to predict motor abilities

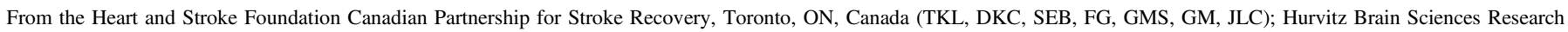

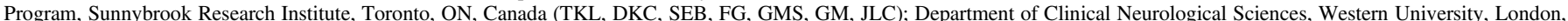

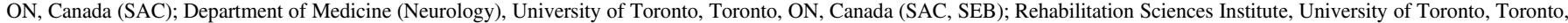

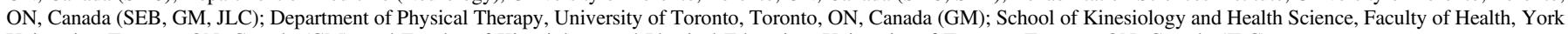
University, Toronto, ON, Canada (GM); and Faculty of Kinesiology and Physical Education, University of Toronto, Toronto, ON, Canada (JLC)

Received November 25, 2019. Final Revisions Submitted April 28, 2020. Date of Acceptance May 29, 2020.

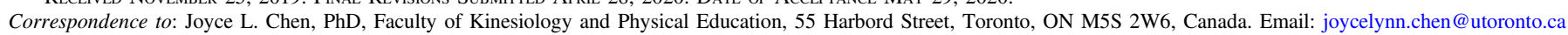


post-stroke. While clinical measures alone are sufficient to predict motor recovery in individuals with mild-moderate stroke, the use of TMS to assess CST damage was necessary to predict motor recovery in more severely affected individuals. ${ }^{14}$ Furthermore, the ability to predict outcome reduced length of stay for stroke survivors in acute care and rehabilitation by 1 week. ${ }^{15}$ Despite the predictive ability of these algorithms, technologies such as TMS and MRI may not be readily available for clinical use.

The CST is one of the main motor output pathways in humans, which connects the primary motor cortex (M1) with the spinal cord. Damage to the CST leads to motor deficits. There are two broad approaches to quantify CST damage: (1) fractional anisotropy (FA) and (2) CST-lesion overlap. FA is derived from diffusion-weighted MRI scans and measures the diffusion of water molecules along white-matter tracts, ${ }^{16}$ with lower FA values indicating loss in white-matter integrity. Individuals with stroke with lower FA values of the CST have worse motor deficits than those with higher FA values of the CST. ${ }^{17}$ The CST-lesion overlap is typically derived from T1-weighted structural MRI scans and can be quantified as the amount of overlap between the stroke lesion and CST, and expressed as a percentage (CST Injury). ${ }^{1,2}$ Individuals with stroke who have greater CST Injury have more motor impairment than individuals who have less CST Injury. ${ }^{5-9,18}$ With respect to recovery, previous research has found that CST Injury is a better predictor of motor recovery than FA values involving the CST. ${ }^{2,12,19}$ Furthermore, CST Injury values acquired during the acute stage explain the variance in motor impairment at 3 months post-stroke. ${ }^{11}$ Taken together, CST Injury is a biomarker of the structural integrity of the motor system and may yield important information for clinical decisions of individuals with stroke.

Traditionally, CST Injury is derived from T1-weighted MRI scans on which the stroke lesion is traced, ${ }^{7-9,12,18}$ although a few studies have used either T2-weighted ${ }^{5}$ or diffusion-weighted ${ }^{6,11}$ MRI scans for lesion tracing. Yet not all individuals with stroke receive an MRI scan. It is estimated that $20 \%-30 \%$ of patients admitted to hospital with acute stroke worldwide do not obtain an MRI scan, ${ }^{20}$ with a couple studies that have reported this percentage in fact to be over $40 \%$ in Germany ${ }^{21}$ and Canada, ${ }^{22}$ due to MRI contraindications and/or unstable medical status of patients. The ability for computed tomography (CT) scanners to acquire brain images quickly and with fewer contraindications than MRI make CT scans more practical for clinical use. Despite the fact that CT, as opposed to MRI, exposes patients to radiation, CT scans are predominantly acquired at many stroke centers, with up to $80 \%$ of patients in the USA obtaining a CT scan during their hospitalization for stroke. ${ }^{23}$ In Canada, for example, CT scans are acquired as part of routine stroke care to rule out bleeds and to determine eligibility for hyperacute therapies. Clinical MRI scans are only acquired if the CT scan is inconclusive or if there are unanswered questions from the CT scan alone. Despite the evidence that CST Injury, as derived from T1-weighted MRI, correlates with motor recovery, ${ }^{2,12}$ it is unclear how this work can translate to practice. This may be especially important for the $20 \%-30 \%$ of patients who only obtain a CT scan, but do not acquire an MRI scan, for their stroke care. Previous research has yet to investigate the feasibility of imaging modalities other than MRI to derive CST Injury. It is currently unknown whether lesions delineated from CT scans can be used to derive CST
Injury values that provide meaningful information regarding an individual's motor status after stroke. The present proof-ofprinciple study serves as a preliminary step in understanding the relationship between CST Injury, using lesions derived from CT scans, and motor impairment. If no significant relationship is found between CST Injury and motor impairment, then this suggests that one may not be able to derive meaningful information about motor outcome post-stroke from CT scans. Conversely, if a significant relationship is found between CST Injury and motor impairment, then further research is warranted to determine if the CST Injury values are comparable to those obtained from MRI scans. As such, future prospective research is required where MRI and CT are obtained in the same individuals to allow for a direct comparison between the two imaging modalities. All participants included in this study had their CT scan within the acute stage (up to 7 days post-stroke). We hypothesize that CST Injury, derived from lesion tracings using CT scans, will correlate with motor impairment after stroke. If such a relationship is determined, future work can determine if CST Injury derived from CT scans predict an individual's motor recovery.

\section{Methods \\ Participants}

The present retrospective study re-analyzes data from Cheung et al. ${ }^{24}$ that is part of the Rehabilitation Affiliates Program. This program recruited people with stroke from four healthcare sites (Baycrest Centre, Grand River Hospital [Freeport Campus], Providence Healthcare, and Sunnybrook Health Sciences Centre) who completed a battery of clinical assessments to assess their outcome after stroke. The study protocol was approved by the four sites where participants were recruited, specifically the Baycrest Research Ethics Board, Tri-Hospital Research Ethics Board at Grand River Hospital, Providence Healthcare Research Ethics Board, and the Sunnybrook Research Ethics Board. Participants gave informed written consent to participate in the study, and for their data to be included in the database for future research use, which were approved by the research ethics boards from each of the four sites. The data from Cheung et al. ${ }^{24}$ involved participants with and without spasticity in the upper limb following their stroke. From these participants, we included those who (1) had a CT scan within the acute stage (up to 7 days) of their stroke $\mathrm{e}^{25}$ and (2) underwent the ChedokeMcMaster Stroke Assessment (CMSA)-Arm and CMSA-Hand motor assessments. ${ }^{26}$ In total, 37 participants were included in our study.

\section{Motor Assessments}

The CMSA-Arm and CMSA-Hand assess motor impairment in the arm and hand, respectively, and has good validity with the Fugl-Meyer Assessment. ${ }^{26}$ The degree of motor impairment is categorized into seven stages, from 1 (flaccid paralysis) to 7 (normal movement). We summed the CMSA-Arm and CMSAHand scores to obtain a single composite measure (CMSAMotor) representative of upper-limb motor impairment for each participant. This summation was performed since we did not intend to dissociate impairments of the arm from the hand. If a participant completed the CMSA-Arm and CMSA-Hand at multiple time points, we used the CMSA scores closest to the date of their CT scan. 


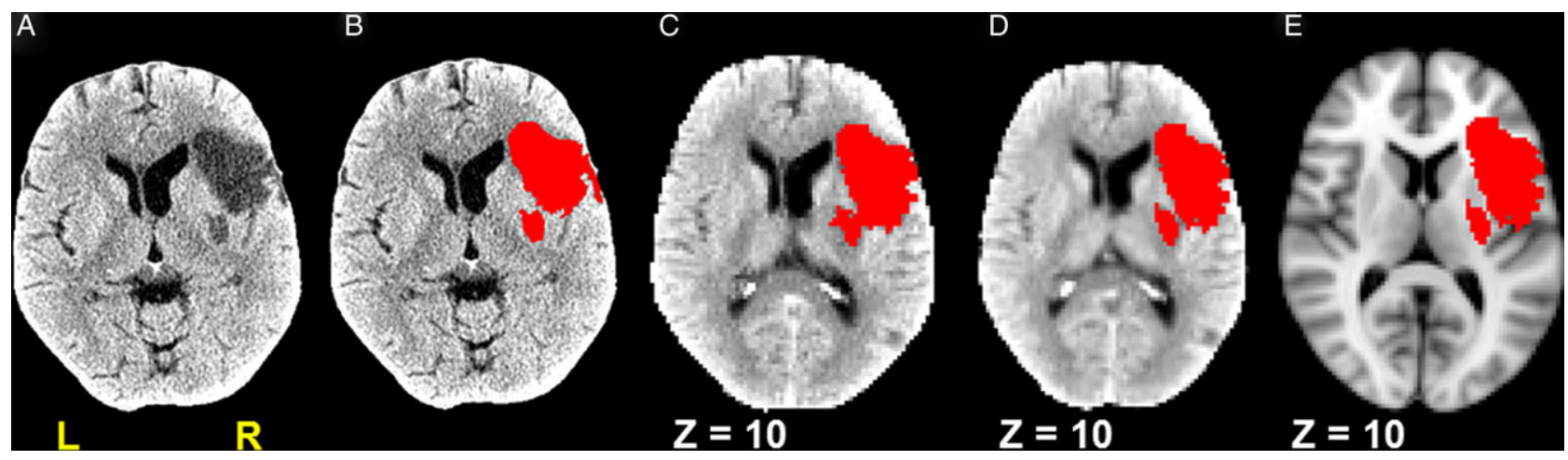

Figure 1: Registration pipeline. (A) The CT brain image of a stroke patient. (B) The CT brain image and stroke lesion tracing (in red). (C) The brain image and stroke lesion in the MNI-ICBM 152 nonlinear $(2 \mathrm{~mm})$ space after linear registration. (D) The brain image and stroke lesion in MNI-ICBM 152 nonlinear (2 mm) space after nonlinear registration. (E) The stroke lesion overlaid on the MNI-ICBM 152 nonlinear (2 mm) template. (" $L$ " represents left; " $R$ " represents right).

\section{CT Scan Parameters}

The clinical CT scans were acquired with the following parameters: in-plane resolution $=0.45 \times 0.45 \mathrm{~mm}^{2}$ or $0.49 \times 0.49 \mathrm{~mm}^{2}$; slice thickness $=5 \mathrm{~mm} ; 24-32$ axial slices. The clinical CT scans were de-identified prior to being included in the database, and hence further details regarding scanner site or model could not be retrieved. It is important to note, however, that various clinical $\mathrm{CT}$ scanners were used to acquire the imaging data. Thus, the CT scan parameters were heterogeneous within and across healthcare sites where the data were collected.

\section{Data Analyses}

\section{Stroke Lesion}

The lesions were manually traced on the CT scans using the imaging software ITK-SNAP (University of Pennsylvania, Philadelphia, PA, USA). ${ }^{27}$ We included participants with CT scans acquired during the hyperacute and acute stages ( $0-1$ day) since previous research has reported that ischemic strokes can be detected as early as $3^{28}$ or $6^{29}$ hours after stroke onset. Study authors (DKC and SAC) used radiology reports to guide their lesion tracings but were blinded to the study objectives and clinical status of each participant. All lesion tracings were reviewed by an experienced research radiologist (FG). The lesion tracings were used to create a binary lesion mask for each participant. The lesion mask was used to exclude lesioned brain regions in the CT-to-MRI registration procedure and for the calculation of CST Injury (both processes described below).

\section{Preprocessing}

We removed non-brain tissue (i.e., skull) in the CT volumes using Analyze10 (AnalyzeDirect Inc., Overland Park, KS, USA). For each slice, we used semi-automated seeding and manually set the image intensity threshold range to distinguish between brain and skull. The CT volumes without the skull were subsequently applied in the registration and analysis procedures described below.

\section{CT-to-MRI Registration}

We performed a two-step registration procedure to transform the participant's brain volume in CT space to standard MRI space. Here, we defined MRI space as the Montreal Neurological Institute-International Consortium of Brain Mapping (MNIICBM) 152 nonlinear $(2 \mathrm{~mm}$ ) template, which is a template of the human brain acquired from T1-weighted MRI scans averaged across 152 individuals. ${ }^{30}$ In the first step, we registered the CT volumes to the MNI-ICBM 152 nonlinear $(2 \mathrm{~mm})$ space with an affine transformation using FMRIB's Linear Image Registration Tool. ${ }^{31}$ To register images from two different imaging modalities (i.e., CT and MRI space), we applied the correlation ratio cost function. ${ }^{32}$ The output from this step was applied in the second step, which involved nonlinear registration to the MNI-ICBM 152 nonlinear $(2 \mathrm{~mm})$ space using registration software from Advanced Normalization Tools $1.9 \mathrm{v} 4 .{ }^{33,34}$ In both steps, the lesion mask for each participant was applied to exclude the lesion areas during the registration procedure. Figure 1 summarizes the two-step registration pipeline that was applied to register the CT brain images to MNI space.

\section{Calculation of CST Injury}

All analyses were performed using FMRIB Software Library $(\text { FSL })^{31}$ version 5.0. CST Injury was defined as the amount of overlap between the stroke lesion of the participant and a CST template, expressed as a percentage. The lesion mask for each participant was registered to the MNI-ICBM 152 nonlinear $(2 \mathrm{~mm})$ space using the two-step procedure described previously. Lesion masks were visually inspected to ensure accuracy in lesion location with respect to the lesion tracings in CT space, after registration to the MNI-ICBM 152 nonlinear $(2 \mathrm{~mm})$ template. Lesion masks were flipped along the mid-sagittal plane, if necessary, for all lesions to be displayed on the right hemisphere.

To derive the CST template, we obtained a binary mask of the right M1-CST, with no threshold applied, using the CST template provided by the Johns Hopkins University (JHU) white-matter tractography atlas, ${ }^{35}$ available in FSL. ${ }^{31}$ Based on our prior work ${ }^{18}$ and the method first described by Pineiro et al., ${ }^{5}$ we determined the 


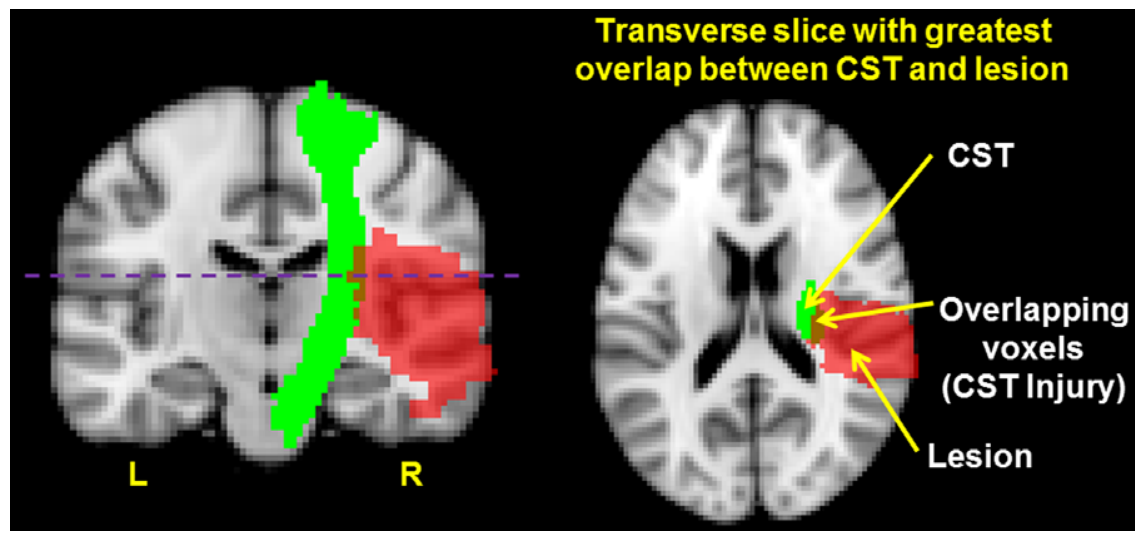

Figure 2: A schematic depicting the CST Injury calculation. The transverse slice of the CST template (green) from the JHU white-matter tractography atlas with the greatest overlap with the stroke lesion (red) was used to determine CST Injury. The purple dotted line represents the transverse slice in which the CST Injury calculation is derived since this slice has the greatest overlap between the CST and stroke lesion. (" $L$ " represents left; " $R$ " represents right).

transverse slice of the CST with the greatest overlap with the lesion (Figure 2) and performed the following calculation:

$$
\begin{gathered}
\text { CST Injury }= \\
\left(\frac{\text { Number of overlapping voxels between the CST and lesion for the transverse slice }}{\text { Total number of CST voxels for the transverse slice }}\right) \\
\times 100 \%
\end{gathered}
$$

This equation was applied to the stroke lesion tracing for each participant. CST Injury ranges from 0\% (no overlap between CST template and lesion for all transverse slices of the CST template) to $100 \%$ (full overlap between CST template and lesion for at least one transverse slice of the CST template). A single transverse slice with the greatest overlap with the lesion can be representative of injury severity to the CST since damage to this slice leads to compromised tract integrity of CST fibers above and below. We also used this equation because it takes into consideration the participant's lesion location. The transverse slices of the CST composed of fewer voxels represent the bottleneck of the tract since a greater proportion of CST fibers pass through a smaller cross-sectional area, relative to other CST slices. Furthermore, the defined range of CST Injury values (0\%-100\%) allows one to more easily compare the severity of injury to the CST across participants.

\section{Secondary Analyses}

We also examined the influence of the type of CST template and approach to calculate CST-lesion overlap on the relationship between CST Injury and CMSA-Motor score. To determine whether CST Injury is influenced by the specificity of the CST template, we calculated CST Injury values using a binary mask of the right M1-CST, with no threshold applied, from the sensorimotor area tract template (SMATT) ${ }^{36}$ The M1-CST mask from the SMATT was registered to the MNI-ICBM 152 nonlinear $(2 \mathrm{~mm})$ template and then used as the CST template to calculate CST Injury. The SMATT-CST template is comprised of CST voxels that pass through M1, the posterior limb of internal capsule, and the cerebral peduncle. We used this template since it minimized overlap of fibers that pass through other sensorimotor regions, such as premotor cortex and supplementary motor area, hence increasing tract specificity. The JHU-CST template has approximately six times more voxels (6606 voxels) than the SMATT-CST template (1083 voxels) (Supplementary Figure S1).

There are multiple approaches to calculate the amount of CST-lesion overlap. ${ }^{6-9}$ To determine whether our results were influenced by the CST-lesion overlap approach, we also calculated weighted CST lesion load (wCST-LL), based on the method first described by Zhu et al. ${ }^{7}$ This approach calculates the volume of overlap between the CST and lesion and, hence, includes all slices with overlapping voxels, as opposed to a single representative slice. Furthermore, this approach considers the probability that the overlapping voxel is found in the CST of healthy individuals and the cross-sectional area of the tract for which the overlapping voxel is located. Aside from the two CSTlesion overlap methods used here, various approaches can be applied to CT scans, but it is beyond the scope of the present study to compare each CST-lesion overlap method.

Taken together, the secondary analyses in the present study involve one or both of the following variables: (1) type of CST template (JHU-CST and SMATT-CST) and (2) approach to calculate CST-lesion overlap (CST Injury and wCST-LL). The secondary analyses allow us to determine whether our findings can be replicated in our dataset, when using different CST templates and/or approaches to calculate CST-lesion overlap.

\section{Statistical Analyses}

To determine the variables that explain the variability in motor impairment, we performed a stepwise regression analysis. In this analysis, a model was built from a pool of predictor variables using the following criteria: (1) the probability of the $F$-statistic for a variable to enter the model is $p<0.05$ and (2) the probability of the $F$-statistic for a variable to be removed from the model is $p>0.10$. The pool of predictor variables available to explain the variability in CMSA-Motor score consisted of: CST Injury and seven covariates of participant demographics (age, sex, lesion volume, presence/absence of spasticity, stroke-to-scan time, time between $\mathrm{CT}$ scan and motor assessment, and hemisphere in which the stroke occurred). Given that there may be up to seven covariates to significantly explain variability in 
Table 1: Participant demographics and performance on motor assessments

\begin{tabular}{|c|c|}
\hline Participants & 37 \\
\hline \multicolumn{2}{|c|}{ Demographics } \\
\hline Age (years) & $66.9 \pm 12.7(43-98)$ \\
\hline \multicolumn{2}{|l|}{ Sex } \\
\hline Male & $23(62 \%)$ \\
\hline Female & $14(38 \%)$ \\
\hline Stroke-to-scan time (days) & $2.5 \pm 1.9(0-7)$ \\
\hline \multicolumn{2}{|l|}{ Lesion location } \\
\hline Left hemisphere & $20(54 \%)$ \\
\hline Right hemisphere & $17(46 \%)$ \\
\hline Time between scan and assessment (days) & $71.6 \pm 80.9(7-370)$ \\
\hline \multicolumn{2}{|c|}{ Neurological measures } \\
\hline \multicolumn{2}{|l|}{ CST Injury (\%) } \\
\hline JHU-CST Injury & $49.6 \pm 38.1(0-100)$ \\
\hline SMATT-CST Injury & $61.9 \pm 42.2(0-100)$ \\
\hline \multicolumn{2}{|l|}{ Weighted CST lesion load $\left(\mathrm{cm}^{3}\right)$} \\
\hline JHU-wCST-LL & $1.4 \pm 1.3(0-4.8)$ \\
\hline SMATT-wCST-LL & $1.5 \pm 1.5(0-5.1)$ \\
\hline \multicolumn{2}{|c|}{ Motor assessments } \\
\hline \multicolumn{2}{|l|}{ Chedoke-McMaster Stroke Assessment } \\
\hline Stage of arm impairment & $4(1-7)$ \\
\hline Stage of hand impairment & $4(1-7)$ \\
\hline Total motor impairment & $8(2-14)$ \\
\hline
\end{tabular}

Summary statistics are presented as mean \pm standard deviation (range) for continuous variables, number of participants (percent of sample) for categorical (i.e., binary) variables, and median (range) for ordinal variables. JHU-CST Injury (percent injury to the JHU CST); SMATT-CST Injury (percent injury to the SMATT CST); JHU-wCST-LL (weighted lesion load to the JHU CST); SMATT-wCST-LL (weighted lesion load to the SMATT CST).

CMSA-Motor score, a stepwise regression approach was ideal since the variables included in the analysis are selected from statistical criteria and hence reduces the potential bias in including or excluding certain covariates in the final analysis. For each step in the model, we report the $R^{2}$ and adjusted $R^{2}\left(R^{2}{ }_{\text {adj }}\right)$ for the model and the beta $(\beta)$ values for each term. We also verified that the residuals from the final model were approximately normally distributed using the Shapiro-Wilk statistic $(p<0.05$ indicates that the data are not normally distributed).

A post hoc analysis was performed to compare the top two CST-lesion overlap models, based on those with the highest $R^{2}$ adj and $\beta$ values, to determine whether one CST-lesion overlap method was better at explaining the variability in motor impairment than the other. We computed the Hotelling's $t$-statistic $\left(t_{h}\right)^{37}$ from the correlation between the unstandardized predicted $\left(y^{\prime}\right)$ values from each model. The $y^{\prime}$ values represent the predicted CMSA-Motor scores calculated from the linear equation of the model. The Hotelling's $t$-statistic is a test for dependent correlations (i.e., regression models involving a common variable
(CMSA-Motor) in the same participant sample). If the Hotelling's $t$-statistic is significant $(p<0.05)$, this indicates that one model is better than the other in explaining motor impairment variability. If $p>0.05$, this indicates that one model is not better than the other and suggests that the explained variance from both models are not significantly different from each other. To limit the number of multiple comparisons, the Hotelling's $t$-statistic was only computed between the top two models since this was the main comparison of interest in this study. All statistics were performed using SPSS version 22.0 (IBM Corp., Armonk, NY, USA).

\section{Results}

Participant demographics and performance on the CMSA are summarized in Table 1 (group data) and Supplementary Tables S1 and S2 (individual data). Lesion tracings for each participant were registered to the MNI-ICBM 152 nonlinear $(2 \mathrm{~mm})$ template and depicted in Figure 3.

\section{Relationship Between CST Injury and CMSA-Motor Scores}

From the pool of predictor variables, JHU-CST Injury was the only variable that entered the model to explain variability in CMSA-Motor score $\left(R_{\text {adj }}^{2}=0.12, p=0.02\right)$ (Table 2). No additional steps were required for the complete model. All demographic variables did not meet the statistical criteria for entry into the model. A scatterplot between JHU-CST Injury and CMSAMotor score is depicted in Figure 4 to illustrate the relationship between these variables.

We also performed secondary analyses examining the type of CST template and approach to calculate CST-lesion overlap. The raw and partial Spearman's correlations were also computed for each of these secondary analyses. Overall, the pattern of results remain the same as what we reported between JHU-CST Injury and CMSA-Motor score, despite using the SMATT-CST template and/or wCST-LL approach to calculate CST-lesion overlap. In each case, CST-lesion overlap was the only variable to enter the stepwise regression analysis to explain variability in motor impairment. None of the demographic covariates were included in the final model. Table 3 summarizes the results from the main and secondary regression analyses. Tables of the full regression results and scatterplots for the secondary analyses are in the Supplementary Materials (Supplementary Tables S3 to S5 and Supplementary Figures S2 to S4).

A post hoc analysis was performed to compare the two CST-lesion overlap models with the highest $R^{2}$ adj and $\beta$ values. Of the four CST-lesion overlap models, the two models with the highest $R^{2}$ adj and $\beta$ values were JHU-wCST-LL $\left(R^{2}{ }_{\text {adj }}=\right.$ $0.13 ; \beta=-0.39)$ and SMATT-CST Injury $\left(R_{\text {adj }}^{2}=0.13 ; \beta=-0.39\right)$. The correlation between the unstandardized predicted CMSA-Motor scores in the JHU-wCST-LL model and SMATT-CST Injury model was $r(35)=0.78, p<0.001$. A comparison between these models demonstrated that the explained variance in the JHU-wCST-LL model was not significantly different from the SMATT-CST Injury model $\left(t_{h}(34)=0.01, p=0.99\right)$.

\section{Discussion}

Previous studies have shown that, using TMS and MRI, CST Injury correlates with motor impairment in individuals with 


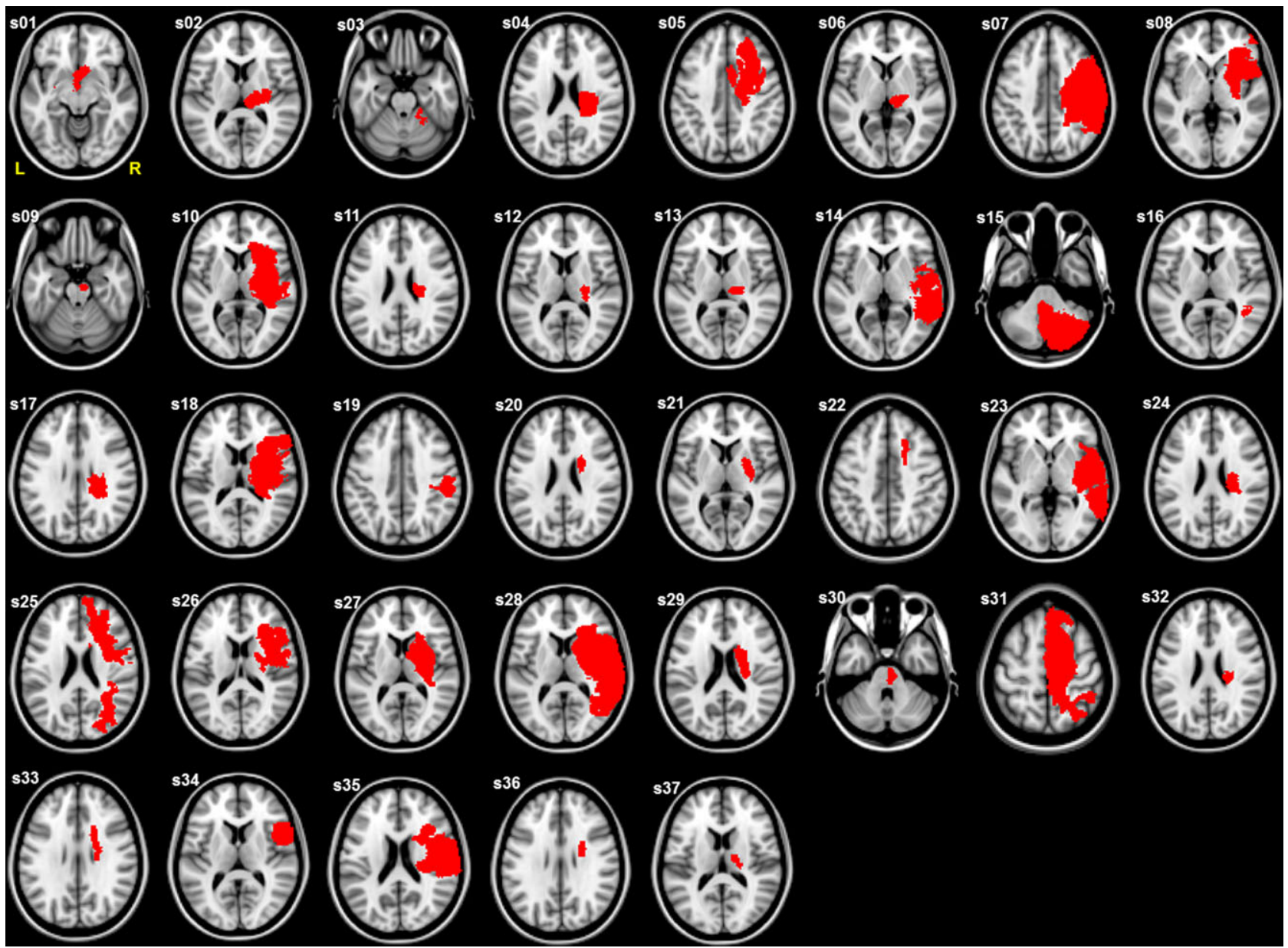

Figure 3: Stroke lesion tracings. Lesions (in red) for each participant are overlaid on the MNI-ICBM 152 nonlinear (2 mm) template. The transverse slice of the lesion with the largest cross-sectional lesion area is displayed (" $s$ " represents subject; " $L$ " represents left; " $R$ " represents right).

stroke. $^{4-11,18}$ In most centers, CT scans, rather than MRI, are acquired during routine clinical acute stroke care. In this proofof-principle study, we examined for the first time the relationship between motor impairment and CST Injury derived from CT scans. The CT scans for each participant were acquired within the acute stage (up to 7 days post-stroke). We found that CST Injury was the only predictor to explain the variability in motor impairment scores in individuals with stroke. None of the participant demographics met the criteria to enter the final model to explain motor impairment variability observed in participants. The alternative CST-lesion overlap approaches (i.e., SMATT-CST Injury, JHU-wCST-LL, and SMATT-wCSTLL) were also the only predictors to enter the final model in the secondary analyses to explain variability in upper-limb motor impairment. A post hoc comparison between the top two CST-lesion overlap approaches (i.e., JHU-wCST-LL and SMATTCST Injury) found that the explained variance in either model was not significantly different from each other. This suggests that the relationship between CST-lesion overlap and motor impairment may not necessarily be influenced by CST template specificity or CST-lesion overlap calculation. The convergence of results between the different approaches suggests that the relationship between CST-lesion overlap, using lesions derived from CT scans, and motor impairment can be replicated. Although we report a significant relationship between CST Injury and motor impairment, it is important to note that the adjusted $R^{2}$ values were modest.

Our results follow the same trend as prior work that used MRI to derive CST Injury. However, we found that CST Injury, derived from CT scans, only explained 12\% variance (adjusted $R^{2}$ ) in motor outcome after stroke. In contrast, Feng et al. ${ }^{11}$ reported that CST Injury, derived from MRI, explains up to $69 \%$ variance in motor outcome at the acute stage. This shows that CT scans are inferior to MRI scans for motor impairment, likely because they do not accurately measure CST Injury.

There are several reasons why our CT scan predictions of CST Injury are sub-optimal. There is variability with respect to how and when the imaging and behavioral data were collected across participants. Various clinical CT scanners were used to acquire the imaging data thereby leading to heterogeneity in the CT scan parameters across participants. The number of axial slices acquired varied across participants, and in some scans, the slice thickness in the base of the skull was different from the rest of the brain. As a result, lesions traced in CT space may not register to the precise location in MRI space which may ultimately lead to a lower correlation between CST Injury and motor impairment. Furthermore, the time between the acquisition of the CT scan and motor assessment varied across participants. We therefore included this variable in the pool of 
Table 2: Stepwise regression for CMSA-Motor using JHU-CST Injury and demographic covariates as predictor variables

\begin{tabular}{|c|c|c|c|c|c|}
\hline & $R^{2}$ & Adjusted $R^{2}$ & $p$-value & $\beta$ & $p$-value \\
\hline Step 1: & 0.14 & 0.12 & $0.02^{*}$ & & \\
\hline \multicolumn{6}{|l|}{ Variables included } \\
\hline JHU-CST Injury & & & & -0.38 & $0.02^{\wedge}$ \\
\hline \multicolumn{6}{|l|}{ Variables excluded } \\
\hline Age & & & & 0.10 & 0.54 \\
\hline Sex & & & & 0.18 & 0.26 \\
\hline Lesion volume & & & & 0.05 & 0.82 \\
\hline Presence/absence of spasticity & & & & -0.12 & 0.48 \\
\hline Stroke-to-scan time & & & & -0.04 & 0.78 \\
\hline $\mathrm{CT}$ scan to motor assessment time & & & & 0.19 & 0.23 \\
\hline Stroke hemisphere & & & & 0.008 & 0.96 \\
\hline
\end{tabular}

$R^{2}$, adjusted $R^{2}, \beta$, and associated $p$-values for the stepwise regression model to explain variability in CMSA Stage of Arm and Hand (CMSA-Motor) score. JHU-CST Injury is the only variable that met statistical criteria (i.e., probability of $F$-statistic is $p<0.05$ ) to enter the model. All demographic covariates did not meet the statistical criteria and hence were excluded from the model.

$* p<0.05$ for the final model.

${ }^{\wedge} p<0.05$ for the $\beta$-value of JHU-CST Injury.

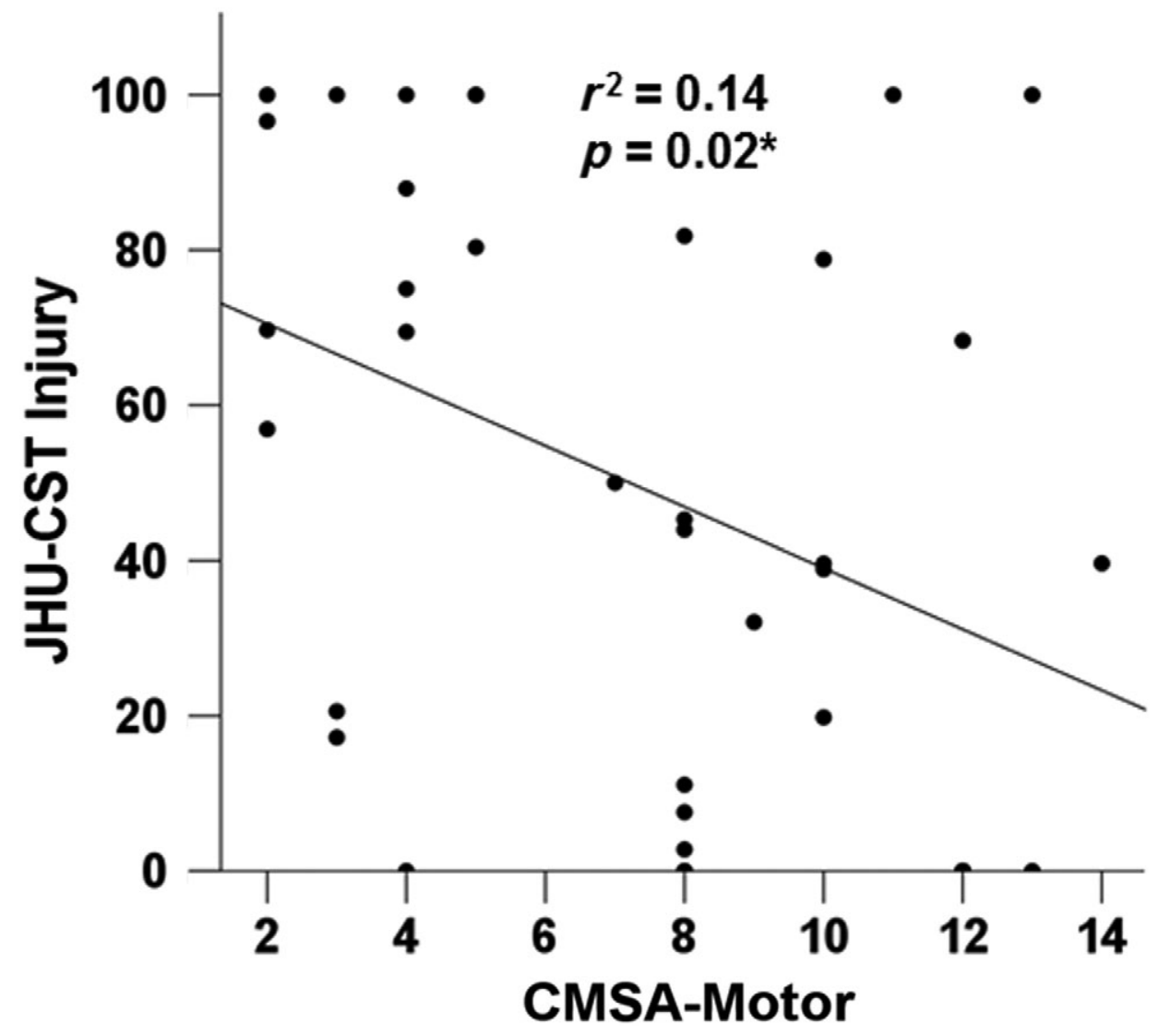

Figure 4: Scatterplot between CST Injury and CMSA-Motor score. The raw correlation is based on CST Injury calculations using the JHU white-matter tractography atlas as the CST template. *Correlation significant at $\mathrm{p}<0.05$. 
Table 3: Summary of stepwise regression results using different CST-lesion overlap approaches to explain variability in CMSAMotor score

\begin{tabular}{l|c|c|c|c|c}
\hline $\begin{array}{l}\text { Variables included in } \\
\text { stepwise regression }\end{array}$ & $\boldsymbol{R}^{\mathbf{2}}$ & Adjusted $\boldsymbol{R}^{\mathbf{2}}$ & $\boldsymbol{p}$-value & $\boldsymbol{\beta}$-value \\
\hline Step 1: Variables included & 0.14 & 0.12 & $0.02^{*}$ & -0.38 \\
\hline JHU-CST Injury & & & & $0.02^{\wedge}$ \\
\hline Step 1: Variables included & 0.15 & 0.13 & $0.02^{*}$ & & \\
\hline JHU-wCST-LL & & & & -0.39 & \\
\hline Step 1: Variables included & 0.15 & 0.13 & $0.02^{*}$ & & $-0.02^{\wedge}$ \\
\hline SMATT-CST Injury & & & & $0.02^{*}$ & \\
\hline Step 1: Variables included & 0.14 & 0.11 & & -0.37 & \\
\hline SMATT-wCST-LL & & & & \\
\hline
\end{tabular}

Demographic covariates did not meet the statistical criteria (i.e., probability of $F$-statistic is $p<0.05$ ) in all cases to enter the model.

$* p<0.05$ for the final model.

${ }^{\wedge} p<0.05$ for the beta $(\beta)$ value of the CST-lesion overlap variable included in the model.

covariates to be considered in the stepwise regression. However, there may be additional changes at the neural or behavioral levels during this period, such as neural plasticity to improve movement, which may influence the relationship between CST Injury and motor impairment. Thus, CST Injury values obtained within the acute stage may not accurately reflect the motor impairment measured at the subacute or chronic stage. On a related note, the wide range in the stroke-to-assessment times across participants also limits the ability for this study to make conclusions about the predictive ability in CT scans on motor outcome for a defined time period after stroke. Second, delineation of the stroke lesion boundaries may be overestimated in CT scans acquired during the hyperacute or acute stages, due to factors such as inflammation or hemorrhagic transformation, ${ }^{25}$ and may lead to inaccurate CST Injury values. In contrast, Feng et al. ${ }^{11}$ used diffusion-weighted MRI scans which provided good distinction between lesion and normal tissue and may allow for differentiation between lesion and edema that would yield more accurate CST Injury values. Third, this was a retrospective study that analyzed a subset of the behavioral and neuroimaging data from the Rehabilitation Affiliates Program, and is likely underpowered. Thus, a prospective study with more participants may be required. Feng et al. ${ }^{11}$ had 67 participants, which is nearly double the number of participants in our proof-of-principle study. The Fugl-Meyer Assessment was not available in the Rehabilitation Affiliates Program from which we acquired our data for this study. Instead, the CMSA was collected as part of the Rehabilitation Affiliates Program. The CMSA is used clinically for stroke rehabilitation in Canada, and hence this assessment was widely used for both clinical and research purposes at the time these data were collected. Future prospective studies should use the Fugl-Meyer Assessment since it is the recommended assessment, according to the Stroke Recovery and Rehabilitation Roundtable. ${ }^{38}$ The CMSA has good construct and concurrent validity with the Fugl-Meyer Assessment. ${ }^{26}$ As a result, both assessments similarly measure motor impairment, ${ }^{26,39}$ and hence the interpretation of our results can be compared with studies that used the Fugl-Meyer Assessment. However, the CMSA-Motor score may not have sufficient granularity to distinguish between participants with varying degrees of motor impairment, thereby reducing the strength of the relationship with CST Injury. The range of possible CMSAMotor scores (2-14) is small, in comparison to the Fugl-Meyer Assessment (from range 0 to 66). Overall, the modest relationship between CST Injury and motor impairment could be attributed to a number of reasons: (1) variability in data collection across participants; (2) delineation of stroke lesion boundaries may be overestimated; (3) small number of participants, and (4) insufficient granularity involving the motor assessment used in this study.

While MRI is superior to CT in delineating infarct boundaries, we believe our results warrant further study. Despite the effectiveness of TMS and MRI to assess CST damage in individuals with stroke, these technologies are not readily available for clinical use. From a theoretical standpoint, the use of CT scans to derive CST Injury holds merit, given its cost-effectiveness and metal compatibility relative to MRI. ${ }^{40}$ Our preliminary results, albeit modest, suggest that clinically available CT scans may have potential to determine CST Injury, although further research is necessary to address the limitations from our study. Future research is required to determine whether CST Injury values obtained from lesions delineated from CT scans are comparable with those obtained from lesions delineated from MRI scans. Given the heterogeneity in demographic and clinical characteristics across participants with stroke, CST Injury can help researchers stratify participants into the appropriate treatment groups and determine those who will likely achieve the intended treatment response. ${ }^{3}$

We show for the first time that CST Injury, using lesions delineated from CT scans, correlates modestly with motor impairment after stroke. Our results suggest that CT scans may be useful in deriving CST Injury values, but more research is necessary to clarify the reliability of these findings.

\section{ACKNOWLEDGEMENTS}

The authors would like to thank Dr. Matthijs van Eede for assistance on the CT-to-MRI registration and Dr. Malcolm A. Binns for input on the statistical analyses. JLC would also like to thank Dr. Jan Scholz for valuable advice on the project. 


\section{FUNDING}

TKL was supported by the Canadian Partnership for Stroke Recovery Start-up Funds.

\section{CONFLict of InTERest}

The authors have no conflicts of interest to disclose.

\section{Statement of Authorship}

TKL and JLC designed the study, interpreted the data, and wrote the manuscript. TKL analyzed the data. DKC and SAC traced the stroke lesions. FG reviewed all lesion tracings. SEB, GMS, and GM assisted in the interpretation of the data. All authors provided feedback and edited the manuscript.

\section{SUPPLEMENTARY MATERIAL}

To view supplementary material for this article, please visit https://doi.org/10.1017/cjn.2020.112

\section{REFERENCES}

1. Burke E, Cramer SC. Biomarkers and predictors of restorative therapy effects after stroke. Curr Neurol Neurosci Rep. 2013;13(2):329.

2. Kim B, Winstein C. Can neurological biomarkers of brain impairment be used to predict poststroke motor recovery? A systematic review. Neurorehabil Neural Repair. 2017;31(1):3-24.

3. Boyd LA, Hayward KS, Ward NS, et al. Biomarkers of stroke recovery: consensus-based core recommendations from the stroke recovery and rehabilitation roundtable. Int J Stroke. 2017;12(5): 480-93.

4. Stinear CM, Barber PA, Smale PR, Coxon JP, Fleming MK, Byblow WD. Functional potential in chronic stroke patients depends on corticospinal tract integrity. Brain. 2007;130(Pt 1): 170-80.

5. Pineiro R, Pendlebury ST, Smth S, et al. Relating MRI changes to motor deficit after ischemic stroke by segmentation of functional motor pathways. Stroke. 2000;31(3):672-9.

6. Sterr A, Shen S, Szameitat AJ, Herron KA. The role of corticospinal tract damage in chronic motor recovery and neurorehabilitation: a pilot study. Neurorehabil Neural Repair. 2010;24(5):413-9.

7. Zhu LL, Lindenberg R, Alexander MP, Schlaug G. Lesion load of the corticospinal tract predicts motor impairment in chronic stroke. Stroke. 2010;41(5):910-5.

8. Riley JD, Le V, Der-Yeghiaian L, et al. Anatomy of stroke injury predicts gains from therapy. Stroke. 2011;42(2):421-6.

9. Carter AR, Patel KR, Astafiev SV, et al. Upstream dysfunction of somatomotor functional connectivity after corticospinal damage in stroke. Neurorehabil Neural Repair. 2012;26(1):7-19.

10. Byblow WD, Stinear CM, Barber PA, Petoe MA, Ackerley SJ. Proportional recovery after stroke depends on corticomotor integrity. Ann Neurol. 2015;78(6):848-59.

11. Feng W, Wang J, Chhatbar PY, et al. Corticospinal tract lesion load: an imaging biomarker for stroke motor outcomes. Ann Neurol. 2015;78(6):860-70.

12. Cassidy JM, Tran G, Quinlan EB, Cramer SC. Neuroimaging identifies patients most likely to respond to a restorative stroke therapy. Stroke. 2018;49(2):433-8.

13. Feldman SJ, Boyd LA, Neva JL, Peters S, Hayward KS. Extraction of corticospinal tract microstructural properties in chronic stroke. J Neurosci Methods. 2018;301:34-42.

14. Stinear CM, Byblow WD, Ackerley SJ, Smith MC, Borges VM, Barber PA. PREP2: a biomarker-based algorithm for predicting upper limb function after stroke. Ann Clin Transl Neuro. 2017; 4(11):811-20.
15. Stinear CM, Byblow WD, Ackerley SJ, Barber PA, Smith MC. Predicting recovery potential for individual stroke patients increases rehabilitation efficiency. Stroke. 2017;48(4):1011-9.

16. Basser PJ, Mattiello J, LeBihan D. MR diffusion tensor spectroscopy and imaging. Biophys J. 1994;66(1):259-67.

17. Puig J, Pedraza S, Blasco G, et al. Wallerian degeneration in the corticospinal tract evaluated by diffusion tensor imaging correlates with motor deficit 30 days after middle cerebral artery ischemic stroke. AJNR Am J Neuroradiol. 2010;31(7): 1324-30.

18. Lam TK, Binns MA, Honjo K, et al. Variability in stroke motor outcome is explained by structural and functional integrity of the motor system. Sci Rep. 2018;8(1):9480.

19. Doughty C, Wang J, Feng W, Hackney D, Pani E, Schlaug G. Detection and predictive value of fractional anisotropy changes of the corticospinal tract in the acute phase of a stroke. Stroke. 2016;47(6):1520-6.

20. von Kummer R. Clinical efficacy of MRI in stroke. In: von Kummer R, Back T. Magnetic resonance imaging in ischemic stroke. Berlin: Springer; 2006. pp. 17-21.

21. Barber PA, Hill MD, Eliasziw, M, et al. Imaging of the brain in acute ischaemic stroke: comparison of computed tomography and magnetic resonance diffusion-weighted imaging. J Neurol Neurosurg Psychiatry. 2005;76(11):1528-33.

22. Schramm P, Schellinger PD, Klotz E, et al. Comparison of perfusion computed tomography and computed tomography angiography source images with perfusion-weighted imaging and diffusionweighted imaging in patients with acute stroke of less than 6 hours' duration. Stroke. 2004;35(7):1652-8.

23. McDonald JS, Fan J, Kallmes DF, Cloft HJ. Pretreatment advanced imaging in patients with stroke treated with IV thrombolysis: evaluation of a multihospital data base. AJNR Am J Neuroradiol. 2014;35(3):478-81.

24. Cheung DK, Climans SA, Black SE, Gao F, Szilagyi G, Mochizuki G. Lesion characteristics of individuals with upper limb spasticity after stroke. Neurorehabil Neural Repair. 2016;30(1):63-70.

25. Bernhardt J, Hayward KS, Kwakkel G, et al. Agreed definitions and a shared vision for new standards in stroke recovery research: the stroke recovery and rehabilitation roundtable taskforce. Int $\mathbf{J}$ Stroke. 2017;12(5):444-50.

26. Gowland C, Stratford P, Ward M, et al. Measuring physical impairment and disability with the Chedoke-McMaster stroke assessment. Stroke. 1993;24(1):58-63.

27. Yushkevich PA, Piven J, Hazlett HC, et al. User-guided 3D active contour segmentation of anatomical structures: significantly improved efficiency and reliability. Neuroimage. 2006; 31(3):1116-28.

28. Barber PA, Demchuk AM, Zhang J, Buchan AM. Validity and reliability of a quantitative computed tomography score in predicting outcome of hyperacute stroke before thrombolytic therapy. ASPECTS study group. Alberta stroke programme early CT score. Lancet. 2000;355(9216):1670-4.

29. Bal S, Bhatia R, Menon BK, et al. Time dependence of reliability of noncontrast computed tomography in comparison to computed tomography angiography source image in acute ischemic stroke. Int J Stroke. 2015;10(1):55-60.

30. Grabner G, Janke AL, Budge MM, Smith D, Pruessner J, Collins DL. Symmetric atlasing and model based segmentation: an application to the hippocampus in older adults. Med Image Comput Comput Assist Interv. 2006;9(Pt 2):58-66.

31. Jenkinson M, Beckmann CF, Behrens TE, Woolrich MW, Smith SM. FSL. Neuroimage. 2012;62(2):782-90.

32. Jenkinson M, Bannister P, Brady M, Smith S. Improved optimization for the robust and accurate linear registration and motion correction of brain images. Neuroimage. 2002;17(2):825-41.

33. Avants BB, Epstein CL, Grossman M, Gee JC. Symmetric diffeomorphic image registration with cross-correlation: evaluating automated labeling of elderly and neurodegenerative brain. Med Image Anal. 2008;12(1):26-41. 
34. Avants BB, Tustison NJ, Song G, Cook PA, Klein A, Gee JC. A reproducible evaluation of ANTs similarity metric performance in brain image registration. Neuroimage. 2011;54(3):2033-44.

35. Hua $\mathrm{K}$, Zhang J, Wakana $\mathrm{S}$, et al. Tract probability maps in stereotaxic spaces: analyses of white matter anatomy and tractspecific quantification. Neuroimage. 2008;39(1):336-47.

36. Archer DB, Vaillancourt DE, Coombes SA. A template and probabilistic atlas of the human sensorimotor tracts using diffusion MRI. Cereb Cortex. 2018;28(5):1685-99.

37. Hotelling, $H$. The selection of variates for use in prediction with some comments on the general problem of nuisance parameters. Ann Math Stat. 1940;11(3):271-83.
38. Kwakkel G, Lannin NA, Borschmann K, et al. Standardized measurement of sensorimotor recovery in stroke trials: consensusbased core recommendations from the stroke recovery and rehabilitation roundtable. Int J Stroke. 2017;12(5):451-61.

39. Gladstone DJ, Danells CJ, Black SE. The fugl-meyer assessment of motor recovery after stroke: a critical review of its measurement properties. Neurorehabil Neural Repair. 2002;16(3):232-40.

40. Powers WJ, Rabinstein AA, Ackerson T, et al. Guidelines for the Early Management of Patients with acute ischemic stroke: a guideline for healthcare professionals from the American heart association/American stroke association. Stroke. 2018;49(3): e46-e110. 